\title{
Existence Results for Second-Order Impulsive Neutral Functional Differential Equations with Nonlocal Conditions
}

\author{
Meili Li and Chunhai Kou \\ Department of Applied Mathematics, Donghua University, Shanghai 201620, China \\ Correspondence should be addressed to Meili Li, stylml@dhu.edu.cn
}

Received 2 September 2009; Accepted 26 October 2009

Recommended by Guang Zhang

The existence of mild solutions for second-order impulsive semilinear neutral functional differential equations with nonlocal conditions in Banach spaces is investigated. The results are obtained by using fractional power of operators and Sadovskii's fixed point theorem.

Copyright (C) 2009 M. Li and C. Kou. This is an open access article distributed under the Creative Commons Attribution License, which permits unrestricted use, distribution, and reproduction in any medium, provided the original work is properly cited.

\section{Introduction}

The study of impulsive functional differential equations is linked to their utility in simulating processes and phenomena subject to short-time perturbations during their evolution. The perturbations are performed discretely and their duration is negligible in comparison with the total duration of the processes. That is why the perturbations are considered to take place "instantaneously" in the form of impulses. The theory of impulsive differential and functional differential equations has been extensively developed; see the monographs of Bainov and Simeonov [1], Lakshmikantham et al. [2], and Samoilenko and Perestyuk [3], where numerous properties of their solutions are studied, and detailed bibliographies are given.

This paper is devoted to extending existing results to second-order differential equations. To be precise, in [4], the authors used Sadovsii's fixed point theorem for a condensing map to establish existence results for first-order impulsive semilinear neutral functional differential inclusions with nonlocal conditions. Here, we obtain existence results for second-order semilinear impulsive differential equations with nonlocal conditions of the form

$$
\begin{aligned}
\frac{d}{d t}\left[x^{\prime}(t)-F\left(t, x\left(h_{1}(t)\right)\right)\right] & =A x(t)+G\left(t, x\left(h_{2}(t)\right)\right), \quad t \in J=[0, b], t \neq t_{k}, \\
\left.\Delta x\right|_{t=t_{k}} & =I_{k}\left(x\left(t_{k}^{-}\right)\right), \quad k=1, \ldots, m,
\end{aligned}
$$




$$
\begin{gathered}
\left.\Delta x^{\prime}\right|_{t=t_{k}}=\bar{I}_{k}\left(x\left(t_{k}^{-}\right)\right), \quad k=1, \ldots, m, \\
x(0)+g(x)=x_{0}, \quad x^{\prime}(0)=\eta,
\end{gathered}
$$

where $A$ is the infinitesimal generator of a strongly continuous cosine family $C(t), t \in R$, of bounded linear operators in X. Also, $0=t_{0}<t_{1}<\cdots<t_{m}<t_{m+1}=b,\left.\Delta x\right|_{t=t_{k}}=$ $x\left(t_{k}^{+}\right)-x\left(t_{k}^{-}\right),\left.\Delta x^{\prime}\right|_{t=t_{k}}=x^{\prime}\left(t_{k}^{+}\right)-x^{\prime}\left(t_{k}^{-}\right)$. Finally, $F, G, g, I_{k}, \bar{I}_{k}(k=1, \ldots, m)$ and $h_{1}, h_{2}$ are given functions to be specified later.

Other results on second order functional differential equations with and without impulsive effect can be founded in the monographs [5-8].

This paper is organized as follows. In Section 2, we recall briefly some basic definitions and lemmas. The existence theorem for (1.1) and its proof are arranged in Section 3. Our approaches are based on Sadovskii's fixed point theorem, and the theory of strongly continuous cosine families.

\section{Preliminaries}

Definition 2.1 (see [9]). A one-parameter family $C(t), t \in R$, of bounded linear operators in the Banach space $X$ is called a strongly continuous cosine family if and only if

(i) $C(s+t)+C(s-t)=2 C(s) C(t)$ for all $s, t \in R$;

(ii) $C(0)=I$;

(iii) $C(t) x$ is strongly continuous in $t$ on $R$ for each fixed $x \in X$.

We define the associated sine family $S(t), t \in R$, by

$$
S(t) x=\int_{0}^{t} C(s) x d s, \quad x \in X, t \in R
$$

We make the following assumption on $A$ :

$\left(H_{1}\right) A$ is the infinitesimal generator of a strongly continuous cosine family $C(t), t \in R$, of bounded linear operators from $X$ into itself.

The infinitesimal generator of a strongly continuous cosine family $C(t), t \in R$ is the operator $A: X \rightarrow X$ defined by

$$
A x=\left.\frac{d^{2}}{d t^{2}} C(t) x\right|_{t=0}, \quad x \in D(A),
$$


where

$$
D(A)=\{x \in X: C(t) x \text { is twice continuously differentiable in } t\} .
$$

We define

$$
E=\{x \in X: C(t) x \text { is once continuously differentiable in } t\} .
$$

Lemma 2.2 (see [9]). If $C(t), t \in R$, be a strongly continuous cosine family in $X$, then

(i) there exist constants $K \geq 1$ and $\omega \geq 0$ so that $\|C(t)\| \leq K e^{\omega|t|}$, for all $t \in R$, and

$$
\left\|S\left(t_{1}\right)-S\left(t_{2}\right)\right\| \leq K\left|\int_{t_{1}}^{t_{2}} e^{\omega|s|} d s\right|, \quad \forall t_{1}, t_{2} \in R
$$

(ii) if $x \in E$, then $S(t) x \in D(A)$ and $(d / d t) C(t) x=A S(t) x$.

It is proved in [10] that for $0 \leq \alpha \leq 1$, the fractional powers $(-A)^{\alpha}$ exist as close linear operator in $X, D\left((-A)^{\alpha}\right) \subset D\left((-A)^{\beta}\right)$, for $0 \leq \beta \leq \alpha \leq 1$, and $(-A)^{\alpha}(-A)^{\beta}=(-A)^{\alpha+\beta}$ for $0 \leq \alpha+\beta \leq 1$.

We assume in addition the following assumption:

$\left(H_{2}\right)$ for $0 \leq \alpha \leq 1,(-A)^{\alpha}$ maps onto $X$ and is $1-1$, so that $D\left((-A)^{\alpha}\right)$ is a Banach space when endowed with the form $\|x\|_{\alpha}=\left\|(-A)^{\alpha} x\right\|, x \in D\left((-A)^{\alpha}\right)$. We denote this Banach space by $X_{\alpha}$. functions:

Denote $J_{0}=\left[0, t_{1}\right], J_{k}=\left(t_{k}, t_{k+1}\right], k=1,2, \ldots, m$. We define the following classes of $P C\left(J, X_{\alpha}\right)=\left\{x: J \rightarrow X_{\alpha}: x_{k} \in C\left(J_{k}, X_{\alpha}\right), k=0,1, \ldots, m\right.$ and there exist $x\left(t_{k}^{+}\right), x\left(t_{k}^{-}\right), k=1, \ldots, m$ with $\left.x\left(t_{k}\right)=x\left(t_{k}^{-}\right)\right\}:$

$P C^{1}\left(J, X_{\alpha}\right)=\left\{x \in P C\left(J, X_{\alpha}\right): x_{k}^{\prime} \in C\left(J_{k}, X_{\alpha}\right), k=0,1, \ldots, m\right.$ and there exist $x^{\prime}\left(t_{k}^{+}\right), \quad x^{\prime}\left(t_{k}^{-}\right), k=1, \ldots, m$ with $\left.x^{\prime}\left(t_{k}\right)=x^{\prime}\left(t_{k}^{-}\right)\right\}$, where $x_{k}$ and $x_{k}^{\prime}$ represent the restriction of $x$ and $x^{\prime}$ to $J_{k}$, respectively, $(k=0, \ldots, m)$, and $\left\|x_{k}\right\|_{J_{k}}=\sup _{s \in J_{k}}\left\|x_{k}(s)\right\|_{\alpha}$.

Obviously, $P C\left(J, X_{\alpha}\right)$ is a Banach space with the norm $\|x\|_{P C}=\max \left\{\left\|x_{k}\right\|_{J_{k}}, k=\right.$ $0, \ldots, m\}$, and $P C^{1}\left(J, X_{\alpha}\right)$ is also a Banach space with the norm $\|x\|_{P C^{1}}=\max \left\{\|x\|_{P C},\left\|x^{\prime}\right\|_{P C}\right\}$.

Definition 2.3. A function $x(\cdot) \in P C^{1}\left(J, X_{\alpha}\right)$ is said to be a mild solution of (1.1) if

(i) $x(0)+g(x)=x_{0}, x^{\prime}(0)=\eta$;

(ii) $\left.\Delta x\right|_{t=t_{k}}=I_{k}\left(x\left(t_{k}^{-}\right)\right), k=1, \ldots, m$;

(iii) $\left.\Delta x^{\prime}\right|_{t=t_{k}}=\bar{I}_{k}\left(x\left(t_{k}^{-}\right)\right), k=1, \ldots, m$; 
(iv) the restriction of $x(\cdot)$ to the interval $J_{k}(k=0, \ldots, m)$ is continuous and the following integral equation is verified:

$$
\begin{aligned}
x(t)= & C(t)\left[x_{0}-g(x)\right]+S(t)\left[\eta-F\left(0, x\left(h_{1}(0)\right)\right)\right]+\int_{0}^{t} C(t-s) F\left(s, x\left(h_{1}(s)\right)\right) d s \\
& +\int_{0}^{t} S(t-s) G\left(s, x\left(h_{2}(s)\right)\right) d s+\sum_{0<t_{k}<t} C\left(t-t_{k}\right) I_{k}\left(x\left(t_{k}^{-}\right)\right) \\
& +\sum_{0<t_{k}<t} S\left(t-t_{k}\right) \bar{I}_{k}\left(x\left(t_{k}^{-}\right)\right), \quad t \in J .
\end{aligned}
$$

For (1.1), we assume that the following hypotheses are satisfied: for some $\alpha \in(0,1)$,

$\left(H_{3}\right)$ there exists a constant $\beta \in(0,1)$ such that $F: J \times X_{\alpha} \rightarrow X_{\beta}$ is a continuous function, and $(-A)^{\beta} F: J \times X_{\alpha} \rightarrow X_{\alpha}$ satisfies the Lipschitz condition, that is, there exists a constant $L>0$ such that

$$
\left\|(-A)^{\beta} F\left(t_{1}, x_{1}\right)-(-A)^{\beta} F\left(t_{2}, x_{2}\right)\right\|_{\alpha} \leq L\left(\left|t_{1}-t_{2}\right|+\left\|x_{1}-x_{2}\right\|_{\alpha}\right),
$$

for any $0 \leq t_{1}, t_{2} \leq b, x_{1}, x_{2} \in X_{\alpha}$. Moreover, there exists a constant $L_{1}>0$ such that the inequality

$$
\left\|(-A)^{\beta} F(t, x)\right\|_{\alpha} \leq L_{1}\left(\|x\|_{\alpha}+1\right)
$$

holds for any $x \in X_{\alpha}$;

$\left(H_{4}\right)$ the function $G: J \times X_{\alpha} \rightarrow X$ satisfies the following conditions:

(i) for each $t \in J$, the function $G(t, \cdot): X_{\alpha} \rightarrow X$ is continuous, and for each $x \in X_{\alpha}$, the function $G(\cdot, x): J \rightarrow X$ is strongly measurable,

(ii) for each positive number $l \in N$, there is a positive function $w_{l} \in L^{1}(J)$ such that

$$
\sup _{\|x\|_{\alpha} \leq l}\|G(t, x)\| \leq w_{l}(t) \quad \text { a.e. on } J, \quad \liminf _{l \rightarrow \infty} \frac{1}{l} \int_{0}^{b} w_{l}(s) d s=r<\infty
$$

where

$$
\|x\|_{\alpha}=\sup _{0 \leq s \leq b}\|x(s)\|_{\alpha}
$$

$\left(H_{5}\right) h_{i} \in C(J, J), i=1,2 . g: P C^{1}\left(J, X_{\alpha}\right) \rightarrow X_{\alpha}$ is continuous and satisfies that 
(i) there exist positive constants $L_{2}$ and $L_{2}^{\prime}$ such that

$$
\|g(u)\|_{\alpha} \leq L_{2}\|u\|_{P C^{1}}+L_{2}^{\prime} \quad \forall u \in P C^{1}\left(J, X_{\alpha}\right),
$$

(ii) $g$ is a completely continuous map;

$\left(H_{6}\right) I_{k}, \bar{I}_{k} \in C\left(X_{\alpha}, X_{\alpha}\right), k=1, \ldots, m$ are all bounded, that is, there exist constants $d_{k}, \bar{d}_{k}, k=1, \ldots, m$, such that $\left\|I_{k}(x)\right\|_{\alpha} \leq d_{k},\left\|\bar{I}_{k}(x)\right\|_{\alpha} \leq \bar{d}_{k}$, for $x \in X_{\alpha}$;

$\left(H_{7}\right) C(t), t \in J$, is completely continuous.

\section{Main Result}

Theorem 3.1. Let $x_{0} \in X_{\alpha}$. If the hypotheses $\left(H_{1}\right)-\left(H_{7}\right)$ are satisfied, then (1.1) has a mild solution provided that

$$
\begin{gathered}
L_{0}:=2 M_{0} L M b<1, \\
M\left(L_{2}+2 M_{0} b L_{1}+b \gamma\right)<1,
\end{gathered}
$$

where

$$
M=\sup \{\|C(t)\|: t \in J\}, \quad M^{\prime}=\sup \left\{\left\|C^{\prime}(t)\right\|: t \in J\right\}, \quad M_{0}=\left\|(-A)^{-\beta}\right\| .
$$

Proof. Consider the space $B=P C^{1}\left(J, X_{\alpha}\right)$ with morm $\|x\|_{P C^{1}}=\max \left\{\|x\|_{P C},\left\|x^{\prime}\right\|_{P C}\right\}$. We should now show that the operator $P$ defined by

$$
\begin{aligned}
(P x)(t)= & C(t)\left[x_{0}-g(x)\right]+S(t)\left[\eta-F\left(0, x\left(h_{1}(0)\right)\right)\right]+\int_{0}^{t} C(t-s) F\left(s, x\left(h_{1}(s)\right)\right) d s \\
& +\int_{0}^{t} S(t-s) G\left(s, x\left(h_{2}(s)\right)\right) d s+\sum_{0<t_{k}<t} C\left(t-t_{k}\right) I_{k}\left(x\left(t_{k}^{-}\right)\right)+\sum_{0<t_{k}<t} S\left(t-t_{k}\right) \bar{I}_{k}\left(x\left(t_{k}^{-}\right)\right)
\end{aligned}
$$

has a fixed point. This fixed point is then a solution of (2.6).

For each positive number $l$, let $B_{l}=\left\{x \in B:\|x(t)\|_{\alpha} \leq l, t \in J\right\}$. Then for each $l, B_{l}$ is clearly a bounded close convex set in B. We claim that there exists a positive integer $l$ such that $P B_{l} \subseteq B_{l}$. If it is not true, then for each positive integer $l$, there is a function $x_{l}(\cdot) \in B_{l}$, but 
$\left(P x_{l}\right)(\cdot) \notin B_{l}$, that is, $\left\|\left(P x_{l}\right)(t)\right\|_{\alpha}>l$ for some $t(l) \in J$, where $t(l)$ denotes $t$ is dependent on $l$. However, on the other hand, we have

$$
\begin{aligned}
l<\left\|\left(P x_{l}\right)(t)\right\|_{\alpha}=\| & C(t)\left[x_{0}-g\left(x_{l}\right)\right]+S(t)\left[\eta-F\left(0, x_{l}\left(h_{1}(0)\right)\right)\right] \\
& +\int_{0}^{t} C(t-s) F\left(s, x_{l}\left(h_{1}(s)\right)\right) d s+\int_{0}^{t} S(t-s) G\left(s, x_{l}\left(h_{2}(s)\right)\right) d s \\
& +\sum_{0<t_{k}<t} C\left(t-t_{k}\right) I_{k}\left(x_{l}\left(t_{k}^{-}\right)\right)+\sum_{0<t_{k}<t} S\left(t-t_{k}\right) \bar{I}_{k}\left(x_{l}\left(t_{k}^{-}\right)\right) \|_{\alpha} \\
\leq & \left\|C(t)\left[x_{0}-g\left(x_{l}\right)\right]\right\|_{\alpha}+\left\|S(t)\left[\eta-(-A)^{-\beta}(-A)^{\beta} F\left(0, x_{l}\left(h_{1}(0)\right)\right)\right]\right\|_{\alpha} \\
& +\left\|\int_{0}^{t} C(t-s)(-A)^{-\beta}(-A)^{\beta} F\left(s, x_{l}\left(h_{1}(s)\right)\right) d s\right\|_{\alpha} \\
& +\left\|\int_{0}^{t} S(t-s) G\left(s, x_{l}\left(h_{2}(s)\right)\right) d s\right\|_{\alpha} \\
& +\sum_{0<t_{k}<t}\left\|C\left(t-t_{k}\right) I_{k}\left(x_{l}\left(t_{k}^{-}\right)\right)\right\|_{\alpha}+\sum_{0<t_{k}<t}\left\|S\left(t-t_{k}\right) \bar{I}_{k}\left(x_{l}\left(t_{k}^{-}\right)\right)\right\|_{\alpha} \\
\leq & M\left[\left\|x_{0}\right\|_{\alpha}+L_{2} l+L_{2}^{\prime}\right]+M b\left[\|\eta\|_{\alpha}+M_{0} L_{1}(l+1)\right] \\
& +M M_{0} b L_{1}(l+1)+M b \int_{0}^{b} w_{l}(s) d s+M \sum_{k=1}^{m} d_{k}+M \sum_{k=1}^{m}\left(b-t_{k}\right) \bar{d}_{k} .
\end{aligned}
$$

Dividing on both sides by $l$ and taking the lower limits as $l \rightarrow+\infty$, we get $M\left(L_{2}+\right.$ $\left.2 M_{0} b L_{1}+b \gamma\right) \geq 1$. This is a contradiction with the formula (3.2). Hence for some positive integer $l, P B_{l} \subseteq B_{l}$.

Next we will show that the operator $P$ has a fixed point on $B_{l}$, which implies that (1.1) has a mild solution. For this purpose, we decompose $P$ as $P=P_{1}+P_{2}$, where the operators $P_{1}, P_{2}$ are defined on $B_{l}$, respectively, by

$$
\begin{aligned}
\left(P_{1} x\right)(t)= & \int_{0}^{t} C(t-s) F\left(s, x\left(h_{1}(s)\right)\right) d s-S(t) F\left(0, x\left(h_{1}(0)\right)\right), \\
\left(P_{2} x\right)(t)= & C(t)\left[x_{0}-g(x)\right]+S(t) \eta+\int_{0}^{t} S(t-s) G\left(s, x\left(h_{2}(s)\right)\right) d s \\
& +\sum_{0<t_{k}<t} C\left(t-t_{k}\right) I_{k}\left(x\left(t_{k}^{-}\right)\right)+\sum_{0<t_{k}<t} S\left(t-t_{k}\right) \bar{I}_{k}\left(x\left(t_{k}^{-}\right)\right),
\end{aligned}
$$


for $t \in J$, and we will verify that $P_{1}$ is a contraction while $P_{2}$ is a completely continuous operator.

To prove that $P_{1}$ is a contraction, we take $x_{1}, x_{2} \in B_{l}$ arbitrarily. Then for each $t \in J$ and by condition $\left(H_{3}\right)$, we have that

$$
\begin{aligned}
\left\|\left(P_{1} x_{1}\right)(t)-\left(P_{1} x_{2}\right)(t)\right\|_{\alpha} \leq & \left\|\int_{0}^{t} C(t-s)\left[F\left(s, x_{1}\left(h_{1}(s)\right)\right)-F\left(s, x_{2}\left(h_{1}(s)\right)\right)\right] d s\right\|_{\alpha} \\
& +\left\|S(t)\left[F\left(0, x_{1}\left(h_{1}(0)\right)\right)-F\left(0, x_{2}\left(h_{1}(0)\right)\right)\right]\right\|_{\alpha} \\
= & \left\|\int_{0}^{t} C(t-s)(-A)^{-\beta}(-A)^{\beta}\left[F\left(s, x_{1}\left(h_{1}(s)\right)\right)-F\left(s, x_{2}\left(h_{1}(s)\right)\right)\right] d s\right\|_{\alpha} \\
& +\left\|S(t)(-A)^{-\beta}\left\{(-A)^{\beta}\left[F\left(0, x_{1}\left(h_{1}(0)\right)\right)-F\left(0, x_{2}\left(h_{1}(0)\right)\right)\right]\right\}\right\|_{\alpha} \\
\leq & 2 M_{0} L M b \sup _{0 \leq s \leq b}\left\|x_{1}(s)-x_{2}(s)\right\|_{\alpha} \\
= & L_{0} \sup _{0 \leq s \leq b}\left\|x_{1}(s)-x_{2}(s)\right\|_{\alpha} .
\end{aligned}
$$

Thus $\left\|P_{1} x_{1}-P_{1} x_{2}\right\|_{\alpha} \leq L_{0}\left\|x_{1}-x_{2}\right\|_{\alpha}$. Therefore, by assumption $0<L_{0}<1$ (see (3.1)), we see that $P_{1}$ is a contraction.

To prove that $P_{2}$ is completely continuous, firstly we prove that $P_{2}$ is continuous on $B_{l}$. Let $x_{n} \rightarrow x_{*}, x_{n} \in B_{l}$, then by $\left(H_{4}\right)(\mathrm{i})$, we have $G\left(s, x_{n}\left(h_{2}(s)\right)\right) \rightarrow G\left(s, x_{*}\left(h_{2}(s)\right)\right), n \rightarrow \infty$. Since $\left\|G\left(s, x_{n}\left(h_{2}(s)\right)\right)-G\left(s, x_{*}\left(h_{2}(s)\right)\right)\right\| \leq 2 w_{l}(s)$, by the dominated convergence theorem, we have

$$
\begin{aligned}
& \left\|P_{2} x_{n}-P_{2} x_{*}\right\|_{P C} \\
& =\sup _{t \in J} \| C(t)\left[g\left(x_{*}\right)-g\left(x_{n}\right)\right]+\int_{0}^{t} S(t-s)\left[G\left(s, x_{n}\left(h_{2}(s)\right)\right)-G\left(s, x_{*}\left(h_{2}(s)\right)\right)\right] d s \\
& +\sum_{0<t_{k}<t} C\left(t-t_{k}\right)\left[I_{k}\left(x_{n}\left(t_{k}^{-}\right)\right)-I_{k}\left(x_{*}\left(t_{k}^{-}\right)\right)\right]+\sum_{0<t_{k}<t} S\left(t-t_{k}\right)\left[\bar{I}_{k}\left(x_{n}\left(t_{k}^{-}\right)\right)-\bar{I}_{k}\left(x_{*}\left(t_{k}^{-}\right)\right)\right] \|_{\alpha} \\
& \leq M\left\|g\left(x_{*}\right)-g\left(x_{n}\right)\right\|_{\alpha}+\int_{0}^{b}\left\|S(t-s)\left[G\left(s, x_{n}\left(h_{2}(s)\right)\right)-G\left(s, x_{*}\left(h_{2}(s)\right)\right)\right]\right\|_{\alpha} d s \\
& +\sum_{k=1}^{m} M\left\|I_{k}\left(x_{n}\left(t_{k}^{-}\right)\right)-I_{k}\left(x_{*}\left(t_{k}^{-}\right)\right)\right\|_{\alpha}
\end{aligned}
$$




$$
\begin{aligned}
& +\sum_{k=1}^{m} M\left(b-t_{k}\right)\left\|\bar{I}_{k}\left(x_{n}\left(t_{k}^{-}\right)\right)-\bar{I}_{k}\left(x_{*}\left(t_{k}^{-}\right)\right)\right\|_{\alpha} \rightarrow 0 \text { as } n \longrightarrow \infty, \\
& \left\|\left(P_{2} x_{n}\right)^{\prime}-\left(P_{2} x_{*}\right)^{\prime}\right\|_{P C} \\
& =\sup _{t \in J} \| C^{\prime}(t)\left[g\left(x_{*}\right)-g\left(x_{n}\right)\right]+\int_{0}^{t} C(t-s)\left[G\left(s, x_{n}\left(h_{2}(s)\right)\right)-G\left(s, x_{*}\left(h_{2}(s)\right)\right)\right] d s \\
& \quad+\sum_{0<t_{k}<t} C^{\prime}\left(t-t_{k}\right)\left[I_{k}\left(x_{n}\left(t_{k}^{-}\right)\right)-I_{k}\left(x_{*}\left(t_{k}^{-}\right)\right)\right] \\
& \quad+\sum_{0<t_{k}<t} C\left(t-t_{k}\right)\left[\bar{I}_{k}\left(x_{n}\left(t_{k}^{-}\right)\right)-\bar{I}_{k}\left(x_{*}\left(t_{k}^{-}\right)\right)\right] \|_{\alpha} \\
& \leq M^{\prime}\left\|g\left(x_{*}\right)-g\left(x_{n}\right)\right\|_{\alpha}+\int_{0}^{b}\left\|C(t-s)\left[G\left(s, x_{n}\left(h_{2}(s)\right)\right)-G\left(s, x_{*}\left(h_{2}(s)\right)\right)\right]\right\|_{\alpha} d s \\
& +\sum_{k=1}^{m} M^{\prime}\left\|I_{k}\left(x_{n}\left(t_{k}^{-}\right)\right)-I_{k}\left(x_{*}\left(t_{k}^{-}\right)\right)\right\|_{\alpha} \\
& +\sum_{k=1}^{m} M\left\|\bar{I}_{k}\left(x_{n}\left(t_{k}^{-}\right)\right)-\bar{I}_{k}\left(x_{*}\left(t_{k}^{-}\right)\right)\right\|_{\alpha} \longrightarrow 0 \text { as } n \longrightarrow \infty .
\end{aligned}
$$

Thus, $P_{2}$ is continuous.

Next, we prove that $\left\{P_{2} x: x \in B_{l}\right\}$ is a family of equicontinuous functions. Let $\tau_{1}, \tau_{2} \in$ $J, \tau_{1}<\tau_{2}$. Then for each $t \in J$, we have

$$
\begin{aligned}
&\left\|\left(P_{2} x\right)\left(\tau_{2}\right)-\left(P_{2} x\right)\left(\tau_{1}\right)\right\|_{\alpha} \\
& \leq\left\|\left[C\left(\tau_{2}\right)-C\left(\tau_{1}\right)\right]\left(x_{0}-g(x)\right)\right\|_{\alpha}+\left\|S\left(\tau_{2}\right) \eta-S\left(\tau_{1}\right) \eta\right\|_{\alpha} \\
&+\left\|\int_{0}^{\tau_{1}}\left[S\left(\tau_{2}-s\right)-S\left(\tau_{1}-s\right)\right] G\left(s, x\left(h_{2}(s)\right)\right) d s\right\|_{\alpha}+\left\|\int_{\tau_{1}}^{\tau_{2}} S\left(\tau_{2}-s\right) G\left(s, x\left(h_{2}(s)\right)\right) d s\right\|_{\alpha} \\
&+\left\|\sum_{0<t_{k}<\tau_{1}}\left[C\left(\tau_{2}-t_{k}\right)-C\left(\tau_{1}-t_{k}\right)\right] I_{k}\left(x\left(t_{k}^{-}\right)\right)\right\|_{\alpha}+\left\|\sum_{\tau_{1} \leq t_{k}<\tau_{2}} C\left(\tau_{2}-t_{k}\right) I_{k}\left(x\left(t_{k}^{-}\right)\right)\right\|_{\alpha} \\
&+\left\|\sum_{0<t_{k}<\tau_{1}}\left[S\left(\tau_{2}-t_{k}\right)-S\left(\tau_{1}-t_{k}\right)\right] \bar{I}_{k}\left(x\left(t_{k}^{-}\right)\right)\right\|_{\alpha}+\left\|\sum_{\tau_{1} \leq t_{k}<\tau_{2}} S\left(\tau_{2}-t_{k}\right) \bar{I}_{k}\left(x\left(t_{k}^{-}\right)\right)\right\|_{\alpha}
\end{aligned}
$$


Discrete Dynamics in Nature and Society

$$
\begin{aligned}
\leq & \left\|\left[C\left(\tau_{2}\right)-C\left(\tau_{1}\right)\right]\left(x_{0}-g(x)\right)\right\|_{\alpha}+\left\|S\left(\tau_{2}\right) \eta-S\left(\tau_{1}\right) \eta\right\|_{\alpha} \\
& +\int_{0}^{\tau_{1}}\left\|\left[S\left(\tau_{2}-s\right)-S\left(\tau_{1}-s\right)\right] G\left(s, x\left(h_{2}(s)\right)\right)\right\|_{\alpha} d s+\left\|\int_{\tau_{1}}^{\tau_{2}} S\left(\tau_{2}-s\right) G\left(s, x\left(h_{2}(s)\right)\right) d s\right\|_{\alpha} \\
& +\sum_{0<t_{k}<\tau_{1}}\left\|C\left(\tau_{2}-t_{k}\right)-C\left(\tau_{1}-t_{k}\right)\right\| d_{k}+\sum_{\tau_{1} \leq t_{k}<\tau_{2}}\left\|C\left(\tau_{2}-t_{k}\right)\right\| d_{k} \\
& +\sum_{0<t_{k}<\tau_{1}}\left\|S\left(\tau_{2}-t_{k}\right)-S\left(\tau_{1}-t_{k}\right)\right\| \bar{d}_{k}+\sum_{\tau_{1} \leq t_{k}<\tau_{2}}\left\|S\left(\tau_{2}-t_{k}\right)\right\| \bar{d}_{k},
\end{aligned}
$$

and similarly

$$
\begin{aligned}
\|\left(P_{2} x\right)^{\prime} & \left(\tau_{2}\right)-\left(P_{2} x\right)^{\prime}\left(\tau_{1}\right) \|_{\alpha} \\
\leq & \left\|\left[C^{\prime}\left(\tau_{2}\right)-C^{\prime}\left(\tau_{1}\right)\right]\left(x_{0}-g(x)\right)\right\|_{\alpha}+\left\|\left[S^{\prime}\left(\tau_{2}\right)-S^{\prime}\left(\tau_{1}\right)\right] \eta\right\|_{\alpha} \\
& +\left\|\int_{0}^{\tau_{1}}\left[C\left(\tau_{2}-s\right)-C\left(\tau_{1}-s\right)\right] G\left(s, x\left(h_{2}(s)\right)\right) d s\right\|_{\alpha}+\left\|\int_{\tau_{1}}^{\tau_{2}} C\left(\tau_{2}-s\right) G\left(s, x\left(h_{2}(s)\right)\right) d s\right\|_{\alpha} \\
& +\left\|\sum_{0<t_{k}<\tau_{1}}\left[C^{\prime}\left(\tau_{2}-t_{k}\right)-C^{\prime}\left(\tau_{1}-t_{k}\right)\right] I_{k}\left(x\left(t_{k}^{-}\right)\right)\right\|_{\alpha}+\left\|\sum_{\tau_{1} \leq t_{k}<\tau_{2}} C^{\prime}\left(\tau_{2}-t_{k}\right) I_{k}\left(x\left(t_{k}^{-}\right)\right)\right\|_{\alpha} \\
& +\left\|\sum_{0<t_{k}<\tau_{1}}\left[S^{\prime}\left(\tau_{2}-t_{k}\right)-S^{\prime}\left(\tau_{1}-t_{k}\right)\right] \bar{I}_{k}\left(x\left(t_{k}^{-}\right)\right)\right\|\left\|_{\alpha}+\right\| \sum_{\tau_{1} \leq t_{k}<\tau_{2}} S^{\prime}\left(\tau_{2}-t_{k}\right) \bar{I}_{k}\left(x\left(t_{k}^{-}\right)\right)\|\|_{\alpha} \\
\leq & \left\|\left[C^{\prime}\left(\tau_{2}\right)-C^{\prime}\left(\tau_{1}\right)\right]\left(x_{0}-g(x)\right)\right\|_{\alpha}+\left\|\left[S^{\prime}\left(\tau_{2}\right)-S^{\prime}\left(\tau_{1}\right)\right] \eta\right\|_{\alpha} \\
& +\int_{0}^{\tau_{1}}\left\|\left[C\left(\tau_{2}-s\right)-C\left(\tau_{1}-s\right)\right] G\left(s, x\left(h_{2}(s)\right)\right)\right\|_{\alpha} d s+\left\|\int_{\tau_{1}}^{\tau_{2}} C\left(\tau_{2}-s\right) G\left(s, x\left(h_{2}(s)\right)\right) d s\right\|_{\alpha} \\
& +\sum_{0<t_{k}<\tau_{1}}\left\|C^{\prime}\left(\tau_{2}-t_{k}\right)-C^{\prime}\left(\tau_{1}-t_{k}\right)\right\| d_{k}+\sum_{\tau_{1} \leq t_{k}<\tau_{2}}\left\|C^{\prime}\left(\tau_{2}-t_{k}\right)\right\| d_{k} \\
& +\sum_{0<t_{k}<\tau_{1}}\left\|S^{\prime}\left(\tau_{2}-t_{k}\right)-S^{\prime}\left(\tau_{1}-t_{k}\right)\right\| \bar{d}_{\mathrm{k}}+\sum_{\tau_{1} \leq t_{k}<\tau_{2}}\left\|S^{\prime}\left(\tau_{2}-t_{k}\right)\right\| \bar{d}_{k} .
\end{aligned}
$$

The right-hand sides are independent of $x \in B_{l}$ and tend to zero as $\tau_{2}-\tau_{1} \rightarrow 0$, since $C(t), S(t), C^{\prime}(t), S^{\prime}(t)$ are uniformly continuous for $t \in J$ and the compactness of $C(t), S(t)$ for $t>0$ implies the continuity in the uniform operator topology.

The compactness of $S(t)$ follows from that of $C(t)$ and Lemma 2.2.

This shows that $P_{2}$ maps $B_{l}$ into a family of equicontinuous functions.

It remains to prove that $V(t)=\left\{\left(P_{2} x\right)(t): x \in B_{l}\right\}$ is relatively compact in $X$. 
Obviously, by condition $\left(H_{5}\right)$ (ii), $V(0)$ is relatively compact in $B$. Let $0<t \leq b$ be fixed and $0<\epsilon<t$. For $x \in B_{l}$, we define

$$
\begin{aligned}
c c\left(P_{2, \epsilon} x\right)(t)= & C(t)\left[x_{0}-g(x)\right]+S(t) \eta+\int_{0}^{t-\epsilon} S(t-s) G\left(s, x\left(h_{2}(s)\right)\right) d s \\
& +\sum_{0<t_{k}<t} C\left(t-t_{k}\right) I_{k}\left(x\left(t_{k}^{-}\right)\right)+\sum_{0<t_{k}<t} S\left(t-t_{k}\right) \bar{I}_{k}\left(x\left(t_{k}^{-}\right)\right) .
\end{aligned}
$$

Since $C(t), S(t)$ are compact operators, the set $V_{\epsilon}(t)=\left\{\left(P_{2, \epsilon} x\right)(t): x \in B_{l}\right\}$ is relatively compact in $B$ for every $\epsilon, 0<\epsilon<t$. Moreover, for every $x \in \mathrm{B}_{l}$, we have

$$
\begin{aligned}
\left\|\left(P_{2} x\right)(t)-\left(P_{2, \epsilon} x\right)(t)\right\|_{\alpha} & =\left\|\int_{t-\epsilon}^{t} S(t-s) G\left(s, x\left(h_{2}(s)\right)\right) d s\right\|_{\alpha} \leq \int_{t-\epsilon}^{t}\|S(t-s)\| w_{l}(s) d s, \\
\left\|\left(P_{2} x\right)^{\prime}(t)-\left(P_{2, \epsilon} x\right)^{\prime}(t)\right\|_{\alpha} & =\left\|\int_{t-\epsilon}^{t} C(t-s) G\left(s, x\left(h_{2}(s)\right)\right) d s\right\|_{\alpha} \leq \int_{t-\epsilon}^{t}\|C(t-s)\| w_{l}(s) d s .
\end{aligned}
$$

Therefore, there are relatively compact sets arbitrarily close to the set $V(t)$. Hence, the set $V(t)$ is relatively compact in $B$.

Thus, by Arzela-Ascoli theorem, $P_{2}$ is a completely continuous operator. Those arguments enable us to conclude that $P=P_{1}+P_{2}$ is a condensing map on $B_{l}$, and by the fixed point theorem of Sadovskii, there exists a fixed point $x(\cdot)$ for $P$ on $B_{l}$. Therefore, the nonlocal Cauchy problem with impulsive effect (1.1) has a mild solution. The proof is completed.

\section{Acknowledgments}

The work of the M. Li is supported by NNSF of China (no. 10971139). The work of C. Kou is supported by NNSF of China (nos. 10701023 and 10971221). The authors would like to thank the anonymous referee for his/her remarks about the evaluation of the original version of the manuscript.

\section{References}

[1] D. D. Bainov and P. S. Simeonov, Systems with Impulse Effect, Stability, Theory and Applications, John Wiley \& Sons, New York, NY, USA, 1989.

[2] V. Lakshmikantham, D. D. Bainov, and P. S. Simeonov, Theory of Impulsive Differential Equations, vol. 6 of Series in Modern Applied Mathematics, World Scientific, Singapore, 1989.

[3] A. M. Samoilenko and N. A. Perestyuk, Impulsive Differential Equations, vol. 14 of World Scientific Series on Nonlinear Science. Series A: Monographs and Treatises, World Scientific, Singapore, 1995.

[4] X. Fu and Y. Cao, "Existence for neutral impulsive differential inclusions with nonlocal conditions," Nonlinear Analysis: Theory, Methods \& Applications, vol. 68, no. 12, pp. 3707-3718, 2008.

[5] K. Balachandran, J. Y. Park, and S. Marshal Anthoni, "Controllability of second order semilinear Volterra integrodifferential systems in Banach spaces," Bulletin of the Korean Mathematical Society, vol. 36, no. 1, pp. 1-13, 1999. 
[6] M. Benchohra, J. Henderson, and S. K. Ntouyas, "Existence results for impulsive multivalued semilinear neutral functional differential inclusions in Banach spaces," Journal of Mathematical Analysis and Applications, vol. 263, no. 2, pp. 763-780, 2001.

[7] H. R. Henríquez and M. Eduardo Hernández, "Approximate controllability of second-order distributed implicit functional systems," Nonlinear Analysis: Theory, Methods E Applications, vol. 70, no. 2, pp. 1023-1039, 2009.

[8] R. Sakthivel, N. I. Mahmudov, and J. H. Kim, “On controllability of second order nonlinear impulsive differential systems," Nonlinear Analysis: Theory, Methods E Applications, vol. 71, no. 1-2, pp. 45-52, 2009.

[9] C. C. Travis and G. F. Webb, "Cosine families and abstract nonlinear second order differential equations," Acta Mathematica Academiae Scientiarum Hungaricae, vol. 32, no. 1-2, pp. 75-96, 1978.

[10] H. O. Fattorini, "Ordinary differential equations in linear topological spaces. I," Journal of Differential Equations, vol. 5, pp. 72-105, 1969. 


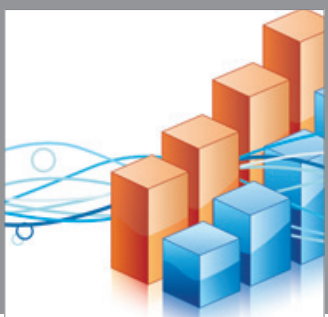

Advances in

Operations Research

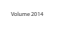

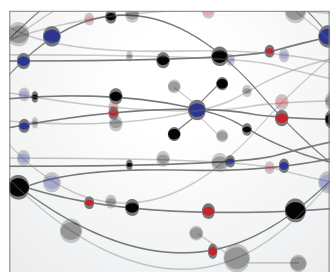

\section{The Scientific} World Journal
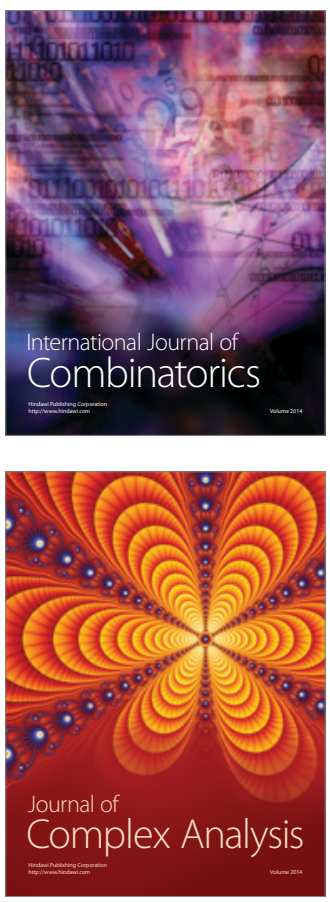

International Journal of

Mathematics and

Mathematical

Sciences
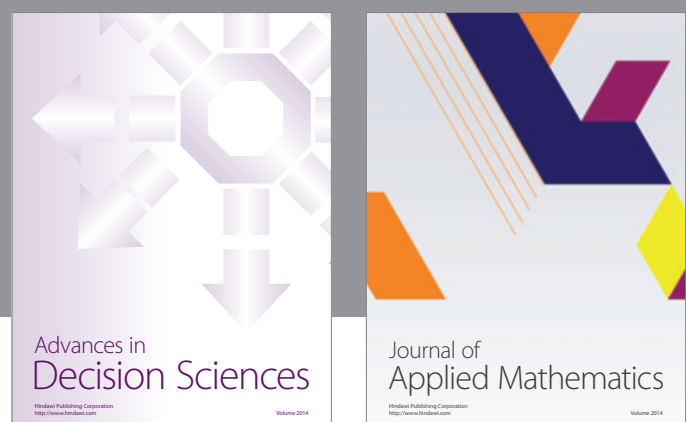

Journal of

Applied Mathematics
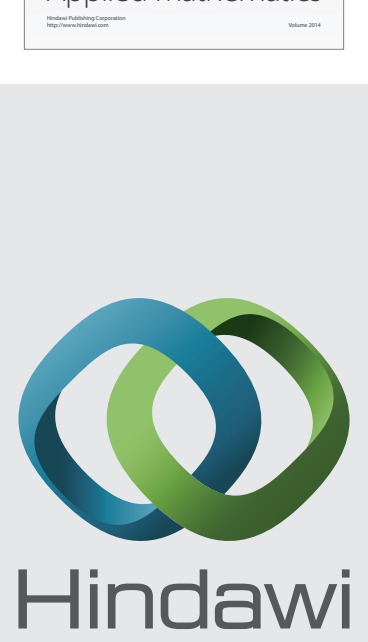

Submit your manuscripts at http://www.hindawi.com
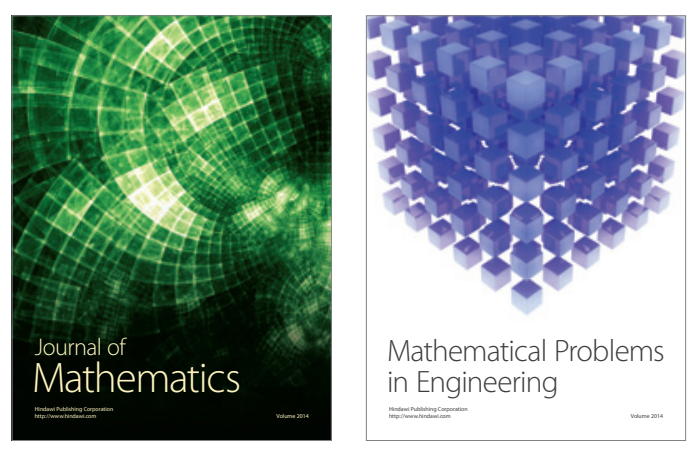

Mathematical Problems in Engineering
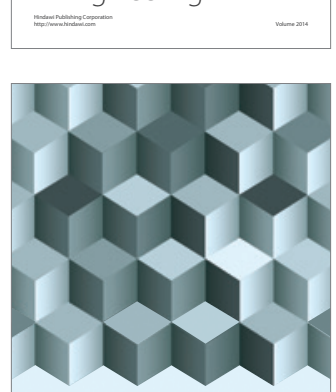

Journal of

Function Spaces
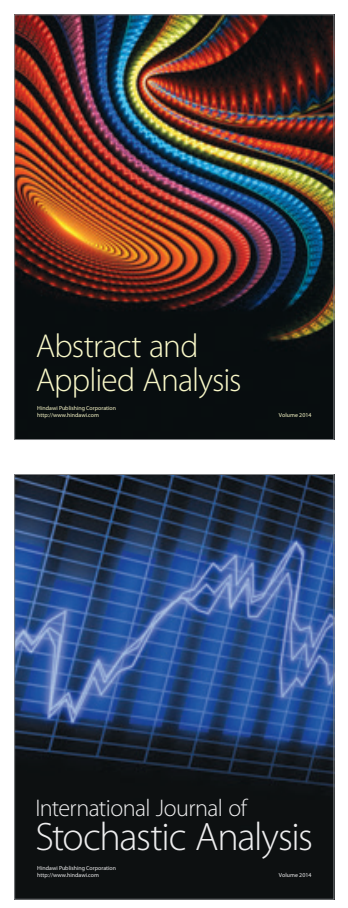

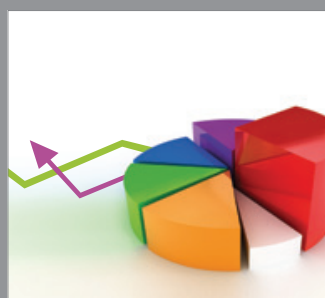

ournal of

Probability and Statistics

Promensencen
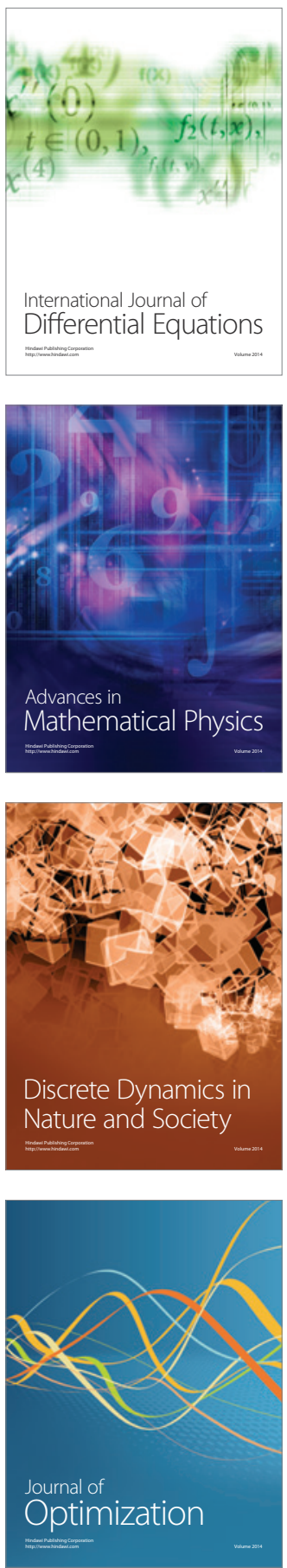